\title{
Confinement and screening in tachyonic matter
}

\author{
F. A. Brito ${ }^{1, a}$, M. L. F. Freire $^{2}$, W. Serafim ${ }^{1,3}$ \\ ${ }^{1}$ Departamento de Física, Universidade Federal de Campina Grande, 58109-970 Campina Grande, Paraíba, Brazil \\ ${ }^{2}$ Departamento de Física, Universidade Estadual da Paraíba, 58109-753 Campina Grande, Paraíba, Brazil \\ ${ }^{3}$ Instituto de Física, Universidade Federal de Alagoas, 57072-970 Maceió, Alagoas, Brazil
}

Received: 26 August 2014 / Accepted: 19 November 2014 / Published online: 20 December 2014

(C) The Author(s) 2014. This article is published with open access at Springerlink.com

\begin{abstract}
In this paper we consider confinement and screening of the electric field. We study the behavior of a static electric field coupled to a dielectric function with the intent of obtaining an electrical confinement similar to what happens with the field of gluons that bind quarks in hadronic matter. For this we use the phenomenon of 'antiscreening' in a medium with exotic dielectric. We show that tachyon matter behaves like in an exotic way whose associated dielectric function modifies the Maxwell equations and affects the fields which results in confining and Coulombianlike potentials in three spatial dimensions. We note that the confining regime coincides with the tachyon condensation, which resembles the effect of confinement due to the condensation of magnetic monopoles. The Coulombian-like regime is developed at large distance, which is associated with a screening phase.
\end{abstract}

\section{Introduction}

The confinement arises naturally in Quantum Chromodynamics (QCD) where the fields of gluons and quarks appear in a confined state at low energies. Similarly to the electric field, we associate the gluon field charges to "color charges". In our studies we show that in general the confining phenomenon can be considered as an effect of a dielectric on the fields and charges with the opposite effect of the screening, called "anti-screening". Throughout the paper we shall mean by anti-screening the way how an electric field behaves in a medium with asymptotic behavior that is opposite to the wellknown asymptotic behavior in a usual dielectric medium. The hadron matter in its confining phase develops this behavior. The tachyonic field is presented here as a good way to develop both anti-screening (the confinement regime) and screening behavior (the Coulomb-potential behavior at sufficiently large interquark separation). This seems to be the

a e-mail: fabrito@df.ufcg.edu.br way in which hadronic matter lives. In three spatial dimensions this effect is represented by Coulomb and confinement potentials describing the potential between quark pairs. Normally the potential of Cornell [1], $v_{c}(r)=-\frac{a}{r}+b r$, is used, where $a$ and $b$ are positive constants, and $r$ is the distance between the heavy quarks. In QED (Quantum Electrodynam$i c s$ ), the effective electrical charge increases when the distance $r$ between a pair of electron-anti-electron decreases. On the other hand, in QCD there is an effect that creates a color charge which decreases as the distance between a pair of quark and anti-quark decreases. Thus, in this sense, QCD develops phenomena effectively opposite to QED. So it is natural to try to understand the QCD phenomena in terms of QED in an "exotic" medium such that it creates an antiscreening effect at some regime near the QCD scale. In this sense we assume that the electric field is now modified by a "dielectric function". Consider $E(r) \equiv \frac{q^{\prime}(r)}{r^{2}} \equiv \frac{q}{G(r) r^{2}}$ where $E(r)$ represents the field due to an electric charge $q$ immersed in a polarizable medium playing the role of a "color" charge coupled to a dielectric function $G(r)$ where $r$ is the distance of the screening (or anti-screening). The action of screening in the QED is expressed according to the effective electrical charge i.e., $q^{\prime}(r \gg d) \leq 1$ or $G(r \gg d) \geq 1$ and $q^{\prime}(r \ll d)>1$ or $G(r \ll d)<1, d$ being the typical size of the screening produced by the polarization of the molecules. On the other hand, in QCD the phenomenon of anti-screening manifests with color charge $q^{\prime}(r \gg R) \gg 1$ or $G(r \gg R) \ll 1$ and $q^{\prime}(r<R) \rightarrow 1$ or $G(r<R) \rightarrow 1$ and $R$ is the radius of the anti-screening which is the typical size of hadrons. Since we are interested in confinement we will focus on the latter case where the dielectric function $G(r)$ is such that the anti-screening provides the QCD color confinement in a pair of heavy quark. In spite of this, in our present study, a dynamical $G(r)$ comes about in the tachyonic matter, such that for sufficiently large distance the anti-screening breaks down and a screening phase associated with hadronization (or light quarks pair creation) takes place. 
Indeed the main proposal in the present study is to define a potential model that may develop appropriate potentials for the confining and screening regimes. Whereas the Cornell potential is good for describing heavy quarks, for light quarks a screening regime occurs for a sufficiently large interquark separation and another appropriate potential is necessary. In our setup the tachyon matter properly develops the desirable confining and screening behaviors. Note that we will continue using Abelian gauge fields as in QED, but now $G(r)$ is properly obtained in order to provide both confinement and screening. We will consider only Abelian projections when these fields are embedded in a color dielectric medium [2,3]. Indeed it is already known that as a result of QCD lattice studies it has been shown that the Abelian part of the string tension accounts for $92 \%$ of the confinement part of the static lattice potential. Thus, it suffices to consider only the Abelian (linear part) of the non-Abelian strength field $[4,5]$. These facts are useful to study the confinement of quarks and gluons inside the hadrons using a phenomenological effective field theory for QCD [1,6-8]. There are different ways to confine the electric field in a dielectric medium with different functions $G(r)$ to obtain confinement. In our case the function $G(r)$ is associated with tachyon condensation $[9,10]$. In general, the behavior of the dielectric function $G$ with respect to $r$ can be governed by a scalar field $\phi(r)$ which describes the dynamics of the dielectric medium in tachyon matter. For this, we use a Lagrangian to describe both the dynamics of gauge and of the scalar fields associated with the tachyon dynamics. The motivation to use this approach is twofold. First, as we shall see later, this a very natural way to obtain a phenomenological effective field theory for QCD since the aforementioned dielectric function is automatically identified with the tachyon potential. As a consequence, this approach may also address the issues of phenomenological aspects of confinement and screening in string theory. Second, since in this setup the electric confinement is related to tachyon condensation, it may bring about new insights on confining supersymmetric gauge theories such as the Seiberg-Witten theory [11-13], which is based on electric-magnetic duality and develops magnetic monopole condensation. We shall consider examples of confinement of the electric field in one and three spatial dimensions.

The paper is organized as follows. In Sect. 2 we briefly review the theory of electromagnetism in a dynamical dielectric medium. In Sect. 3 we introduce the tachyon Lagrangian and potentials. We discuss the solutions obtained in one and three spatial dimensions. In the latter case we find analytical potentials under certain conditions and a numeric solution connecting the confining behavior at small distance to screening behavior at large distance. We explore the physics given by the confining and screening potentials. We show how the tachyon condensation is directly related to the confining phase. In Sect. 4 we present our final considerations.

\section{Maxwell's equations modified by a dielectric function}

In this section we apply the theory of electromagnetism in an exotic dielectric medium to describe the phenomenon of electric confinement. First we write the Maxwell Lagrangian in the vacuum without sources

$\mathcal{L}=-\frac{1}{4} F_{\mu \nu} F^{\mu \nu}$

As is well known the equations of motion for the electromagnetic field are given by

$\partial_{\mu} F^{\mu \nu}=0$

One should note that even in the absence of sources the equations of motion for the electromagnetic field produce spherically symmetric static vacuum solutions (the Coulomb field due to a point charge) [14] as we shall see below.

For electromagnetic fields immersed in a dielectric medium characterized by the dielectric function $G(\phi)$, where $\phi(r)$ is a scalar field that governs the dynamics of the medium, we have the following Lagrangian:

$\mathcal{L}=-\frac{1}{4} G(\phi) F_{\mu \nu} F^{\mu \nu}$

The equations of motion (2) are now described by

$\partial_{\mu}\left[G(\phi) F^{\mu \nu}\right]=0$

where $\mu=0,1,2,3$. Developing the component $v=0$, we simply have

$\nabla \cdot[G(\phi) \mathbf{E}]=0$

We shall be neglecting the magnetic field throughout the paper. This is because the electric field is sufficient for our analysis.

Starting from Eq. (5) we find the electric field $\mathbf{E}$ coupled to the dielectric function $G(\phi)$. Now working on spherical coordinates and assuming that $E(r)$ and $\phi(r)$ are only functions of $r$ and as a consequence $G(\phi)$ follows the same condition, we have the following form:

$\nabla \cdot[G(\phi) \mathbf{E}]=\frac{1}{r^{2}} \frac{\partial}{\partial r}\left(r^{2} G(\phi) E_{r}\right)=0$.

By using this equation we can obtain the vacuum solutions mentioned above. Thus, now we integrate the differential equation to find

$E_{r}=\frac{\lambda}{r^{2} G(\phi)}$. 
Now it is easy to interpret the constant of integration $\lambda=$ $q / 4 \pi \varepsilon_{0}$ to write the Coulomb electric field modified by a dielectric function $G(\phi)$,

$E=\frac{q}{4 \pi \varepsilon_{0} r^{2} G(\phi)}$,

where $E=|\mathbf{E}|=E_{r}$. Therefore, we observe that the dielectric function coupled to the electric field $\mathbf{E}$ changes its magnitude as a function of the radial position $r$.

Let us consider a dielectric function $G(\phi)$ as a function of a dynamical field $\phi$, according to the Lagrangian $[16,17]$

$\mathcal{L}=-\frac{1}{4} G(\phi) F_{\mu \nu} F^{\mu \nu}+\frac{1}{2} \partial_{\mu} \phi \partial^{\mu} \phi-V(\phi)$.

The behavior of the dielectric function $G(\phi)$ will be presented as a consequence of the solutions of the equations of motion obtained by the above Lagrangian. The equations of motion for the electromagnetic field $A_{\mu}$ and the scalar field $\phi$ are given explicitly by the following differential equations:

$\partial_{\mu}\left[G(\phi) F^{\mu \nu}\right]=0$

$\partial_{\mu} \partial^{\mu} \phi+\frac{\partial V(\phi)}{\partial \phi}+\frac{1}{4} \frac{\partial G(\phi)}{\partial \phi} F_{\mu \nu} F^{\mu \nu}=0$.

Then the equations of motion for the dielectric medium and electric field in spherical coordinates are

$\frac{1}{r^{2}} \frac{d}{d r}\left(r^{2} G(\phi) E\right)=0$

$\frac{1}{r^{2}} \frac{d}{d r}\left(r^{2} \frac{d \phi}{d r}\right)=\frac{\partial V}{\partial \phi}-\frac{1}{2} E^{2} \frac{\partial G}{\partial \phi}$.

Based on the previous discussion it is easy to show that the solution of the first equation for the electric field is that given in (8).

To find a confining regime everywhere the dielectric function in (8) must have the following asymptotic behavior:

$$
\begin{array}{lll}
G(\phi(r))=0 & \text { as } & r \rightarrow \infty, \\
G(\phi(r))=1 & \text { as } \quad r \rightarrow 0 .
\end{array}
$$

Particularly, for $G(\phi(\infty)) \sim 1 / r^{2}$, from Eq. (8) we find $E \equiv$ const. This uniform electric field behavior agrees with confinement.

\section{Tachyon condensation and electric confinement}

In this section we discuss the relationship between the phenomena of tachyon condensation and the confinement of the electric field. When we speak of "tachyon" we refer to particles that are faster than light and are associated with instabilities. As magnetic monopoles they have never been observed isolated in nature, although, specially from the superstring point of view, they may always be interacting with other fields or be self-interacting at higher orders to form the tachyon condensation $[9,10,15]$. In our study we show that the electric confinement via tachyon condensation can occur in the same way as the confinement of colorful particles, such as quarks and gluons through condensation of monopoles [11-13].

\subsection{Tachyon Lagrangian with electromagnetic fields}

For simplicity, we first consider the fields depending only on the spatial component $x$, i.e.

$\phi=\phi(x), \quad A_{\mu}=A_{\mu}(x)$.

Thus, the equations of motion discussed in the previous section are now given by

$\frac{d}{d x}[G(\phi) E]=0$,

$-\frac{d^{2} \phi}{d x^{2}}+\frac{\partial V}{\partial \phi}-\frac{1}{2} \frac{\partial G}{\partial \phi} E^{2}=0$,

where we use the fact that $F^{01}=E$. Note that Eq. (17) is a one-dimensional version of Eq. (6). Integrating (17), we have

$G(\phi) E=q \quad \Longrightarrow \quad E=\frac{q}{G(\phi)}$,

so that substituting it into Eq. (18) we find

$-\frac{d^{2} \phi}{d x^{2}}+\frac{\partial V(\phi)}{\partial \phi}-\frac{1}{2} \frac{\partial G(\phi)}{\partial \phi} \frac{q^{2}}{G(\phi)^{2}}=0$

or simply

$-\phi^{\prime \prime}+\frac{\partial V(\phi)}{\partial \phi}-\frac{1}{2} \frac{q^{2}}{G(\phi)^{2}} \frac{\partial G(\phi)}{\partial \phi}=0$.

We note that in the above equation we have, in principle, the potential $V(\phi)$ and the function $G(\phi)$. However, we can restrict these choices considering $G(\phi)=V(\phi)$. As we will see below this choice is legitimate when we are working with a Lagrangian that describes the dynamics of tachyons represented by the scalar field $\phi$.

As is well known from string theory, the dynamics of a tachyonic field $T(x)$ coupled with the electric field $E(x)$ is given by $[9,10,15]$

$$
\begin{aligned}
e^{-1} \mathcal{L} & =-V(T) \sqrt{1-T^{\prime 2}+F_{01} F^{01}} \\
& =-V(T)\left[1-\frac{1}{2}\left(T^{\prime 2}+F_{01} F^{01}\right)+\cdots\right] \\
& \left.=-V(T)+\frac{1}{2} V(T)\left(T^{\prime 2}\right)-\frac{1}{2} V(T) F_{01} F^{01}\right)+\cdots \\
& =-V(\phi)+\frac{1}{2} \phi^{\prime 2}-\frac{1}{2} V(\phi) F_{01} F^{01}+\cdots
\end{aligned}
$$


where $e=\sqrt{|g|}$ in a general spacetime. The power expansion is justified in slow varying tachyon fields, which are suitable to describe tachyon matter $[9,10]$. This derivation remains valid in $3+1$ dimensions for a $\phi$ dependence with purely radial coordinate $r$ which can be identified with $x$.

In the last equation of (22) we use the fact that

$$
\begin{aligned}
V(T(\phi)) & =\left(\frac{\partial \phi}{\partial T}\right)^{2} \Rightarrow \frac{1}{2} V(T)\left(T^{\prime 2}\right) \\
& =\frac{1}{2}\left(\frac{\partial \phi}{\partial T} \frac{\partial T}{\partial x}\right)^{2}=\frac{1}{2} \phi^{\prime 2}
\end{aligned}
$$

where $\phi=f(T)$, or $T=f^{-1}(\phi)$. Note that comparing (22) with (9) we find the equality $G=V$ is legitimate, so that we can write

$$
\begin{aligned}
-\phi^{\prime \prime}+\frac{\partial V}{\partial \phi}-\frac{q^{2}}{2} \frac{1}{V^{2}} \frac{\partial V}{\partial \phi} & =0, \\
-\phi^{\prime \prime}+\frac{\partial V}{\partial \phi}-\frac{q^{2}}{2}\left(\frac{-\partial V^{-1}}{\partial \phi}\right) & =0, \\
\phi^{\prime \prime}-\frac{\partial V}{\partial \phi}-\frac{q^{2}}{2} \frac{\partial V^{-1}}{\partial \phi} & =0, \\
\phi^{\prime \prime}+\frac{\partial}{\partial \phi}\left[-V-\frac{q^{2} V^{-1}}{2}\right] & =0, \\
\phi^{\prime \prime}-\frac{\partial \tilde{V}}{\partial \phi} & =0 .
\end{aligned}
$$

Thus, Eq. (24) is obtained from Eq. (21) using G=V. Note that now we are only left with the equation for the scalar field

$\phi^{\prime \prime}=\frac{\partial \tilde{V}}{\partial \phi}$

with the potential

$\tilde{V}=V+\frac{q^{2}}{2} \frac{1}{V}$.

\subsubsection{Confinement potential for the electric field in a spatial dimension}

For a tachyon Lagrangian in Eq. (22) expanded polynomially, it is explicitly clear that the dielectric function $G(\phi)$ can be equal to the potential $V(\phi)$. Thus, our dielectric function is naturally identified in the context of tachyon theory. Potentials describing the tachyon condensation in string theory are of the type that are zero in the vacuum i.e., when $\phi \rightarrow \pm \phi_{\text {vac }}$ such that $V\left(\phi \rightarrow \pm \phi_{v a c}\right)=0$.

Thus, as an example, we shall adopt the most commonly used tachyon potential of the exponential form

$V=e^{-\alpha \phi}, \quad$ so that $\quad \tilde{V}=2 \cosh (\alpha \phi)$ for $q^{2}=2$. The solution of (24) for this kind of potential is given by

$\phi(x)=\frac{2}{\alpha} \arcsin [\tan (x)]$.

Substituting this solution into the potential $V=e^{-\alpha \phi}$, we find the dielectric function

$G(x)=V(x)=\frac{1}{(\tan (x)+|\sec (x)|)^{2}}$.

Therefore, the model reproduces well the behavior of the electric field confinement

$E(x)=\frac{q}{G(x)}$.

Note that in the limit $x \rightarrow \pm \frac{\pi}{2}$ the electric field diverges ("superconfining" system). On the other hand, in the limit $x \rightarrow 0$, the electric field tends to a constant value $E=q$ (“confining" regime).

\subsubsection{Confinement and Coulomb potential for the electric field in three dimensions}

In three dimensions the extension of Eq. (18) (in the absence of magnetic fields) to radial symmetry is direct and is given by (13), which we recast into the form

$-\left[\frac{1}{r^{2}} \frac{\partial}{\partial r}\left(r^{2} \frac{\partial \phi}{\partial r}\right)\right]+\frac{\partial V}{\partial \phi}-\frac{1}{2} \frac{\partial G}{\partial \phi} E^{2}=0$.

Recalling that the solution for the electric field is given by

$E(r)=\frac{q}{4 \pi \epsilon_{0} G(\phi) r^{2}}$

and substituting into (31) we find

$-\left[\frac{1}{r^{2}} \frac{\partial}{\partial r}\left(r^{2} \frac{\partial \phi}{\partial r}\right)\right]+\frac{\partial V}{\partial \phi}-\frac{1}{2} \frac{\partial G}{\partial \phi}\left[\frac{q}{4 \pi \epsilon_{0} G(\phi) r^{2}}\right]^{2}=0$.

Now considering the fact that $G(\phi)=V(\phi)$ and

$\lambda=\frac{q}{4 \pi \epsilon_{0}}$,

we have

$$
-\left[\frac{1}{r^{2}} \frac{\partial}{\partial r}\left(r^{2} \frac{\partial \phi}{\partial r}\right)\right]+\frac{\partial V(\phi)}{\partial \phi}-\frac{\lambda^{2}}{2} \frac{\partial V(\phi)}{\partial \phi} \frac{1}{r^{4} V(\phi)^{2}}=0 .
$$


Assuming again the exponential tachyon potential $V(\phi(r))=e^{-\alpha \phi(r)}$ we have

$\frac{1}{r^{2}} \frac{\partial}{\partial r}\left(r^{2} \frac{\partial \phi(r)}{\partial r}\right)=\frac{\partial}{\partial \phi(r)}\left[e^{-\alpha \phi(r)}-\frac{\lambda^{2}}{2} \frac{e^{\alpha \phi(r)}}{r^{4}}\right]$.

Since $\phi$ depends only on $r$, we can write our equation in terms of ordinary derivatives to simply obtain

$\frac{d^{2} \phi(r)}{d r^{2}}+\frac{2}{r} \frac{d \phi(r)}{d r}=-\alpha e^{-\alpha \phi(r)}+\frac{\lambda^{2}}{2} \alpha e^{\alpha \phi(r)} \frac{1}{r^{4}}$.

For $\alpha \phi(r)$ sufficiently large (which will easily be satisfied for a large rate $r / r_{\phi}$ - see below), we can compare our result with the results of Refs. [18,19] to the confinement of quarks and gluons with $N_{c}$ colors, i.e.,

$$
\begin{aligned}
& \frac{d^{2} \phi(r)}{d r^{2}}+\frac{2}{r} \frac{d \phi(r)}{d r} \\
& =-\frac{g^{2}}{64 \pi^{2} f_{\phi}}\left(1-\frac{1}{N_{c}}\right) \exp \left(-\frac{\phi(r)}{f_{\phi}}\right) \frac{1}{r^{4}},
\end{aligned}
$$

because the first term on the right side of Eq. (37) becomes negligible. This now allows us to identify our electric charge $q$ in terms of the color charge of quarks and gluons $g$ as follows:

$-\frac{g^{2}}{64 \pi^{2} f_{\phi}}\left(1-\frac{1}{N_{c}}\right)=\alpha \frac{\lambda^{2}}{2}$.

Recall that from Eq. (34) that we have a relation between $\lambda$ and $q$. According to $[18,19]$, the confining solution is given by

$\phi(r)=2 f_{\phi} \ln \left(\frac{r_{\phi}}{r}\right)$.

Manipulating Eq. (39) we have

$-\frac{1}{f_{\phi}}=\alpha \frac{\lambda^{2}}{2} \frac{64 \pi^{2}}{g^{2}}\left(\frac{N_{c}}{N_{c}-1}\right)$.

Now identifying $\alpha=-\frac{1}{f_{\phi}}$, we find

$\lambda=\frac{g}{4 \pi} \sqrt{\frac{N_{c}-1}{N_{c}}}$,

where we have redefined $g \rightarrow g / \sqrt{2}$. Using Eq. (34) we can now write

$q=\epsilon_{0} g \sqrt{\frac{N_{c}-1}{N_{c}}}$.
Substituting (43) into (32), with $G(\phi(r)) \equiv G(r)$, we have

$E(r)=\frac{g}{4 \pi G(r) r^{2}} \sqrt{\frac{N_{c}-1}{N_{c}}}$

where $G(\phi(r))=V(\phi(r))=\exp (-\alpha \phi(r)) ; \phi(r)$ is the confining solution given in Eq. (40). Now using this solution and $\alpha=-\frac{1}{f_{\phi}}$ we arrive at

$G(\phi(r))=V(\phi(r))=\exp \left(2 \ln \frac{r_{\phi}}{r}\right)$

or simply

$G(r)=\left(\frac{r_{\phi}}{r}\right)^{2}$

Substituting Eq. (46) into Eq. (44), we find the electric field modified by a "color dielectric function" $G$ developing a 'confining medium' which means a confining phase given by

$E(r)=\frac{g}{4 \pi r_{\phi}^{2}} \sqrt{1-\frac{1}{N_{c}}}$.

This solution was first obtained in $[18,19]$ in theories with dilatonic solutions [14]. The electric field is constant and therefore implies that the lines of force keep together all the time, which features a flux tube, a confinement due to an "anti-dielectric medium" developed by the hadronic matter that here is represented by 'tachyonic matter'. See discussion below.

\section{- Confining potential}

Integrating (47) we find the confining potential

$v_{c}(r)=\frac{g}{4 \pi r_{\phi}^{2}} \sqrt{1-\frac{1}{N_{c}}} r+c$,

which is a linear confining potential,

$v_{c}(r)=\sigma r+c$,

where $c$ is an integration constant and $\sigma$ is the QCD string tension (which in general also depends on $r$ ), which breaks at some scale of QCD favoring the production of pairs of mesons (hadronization). As we will see, after this scale appears the regime in which a Coulomb-like type potential approaches a constant. Interestingly, our numerical solution to this problem exhibits this behavior.

The electric potential energy between two punctual charges is simply given in terms of the potential and the charge,

$u_{c}(r)=q v_{c}(r)$ 
that is,

$u_{c}(r)=\epsilon_{0} g^{2} \frac{r}{4 \pi r_{\phi}^{2}}\left(1-\frac{1}{N_{c}}\right)$

where for simplicity we have disregarded the integration constant and in Eq. (51), Eq. (43) has been used. So we have a linear confinement type that describes the confinement of quarks and gluons. Note that our treatment is mainly based on an Abelian gauge theory and therefore does not have all the degrees of freedom adequate to describe the colors of gluons. However, as the very expression shows, in the limit $N_{c} \rightarrow \infty$ the electric charge is identical to the color charge. This is the 't Hooft limit (planar limit) where several Feynman diagrams disappear and make the non-Abelian theory simpler. Thus we can understand our approach as an Abelian approach to approximately describe the non-Abelian theory of QCD—the same was considered in $[18,19]$.

\section{- Coulombian potential}

Let us find the Coulomb potential starting from the electric field of Eq. (44), based on the non-confining solution of Eq. (38) for the scalar field [18,19]

$\phi(r)=2 f_{\phi} \ln \left(\frac{r+r_{\phi}}{r}\right)$,

and we have

$G(\phi(r))=V(\phi(r))=G(r)=\left(\frac{r+r_{\phi}}{r}\right)^{2}$.

Recall that $f_{\phi}=-1 / \alpha$ is used in Eq. (53). Substituting this result into Eq. (44) we find

$E(r)=\frac{g}{4 \pi\left(r+r_{\phi}\right)^{2}} \sqrt{1-\frac{1}{N_{c}}}$,

which is a Coulomb potential regularized at distances $r_{\phi}$. Integrating the field as a function of $r$ we obtain the 'Coulomb' potential as follows:

$v_{c l}(r)=-\frac{g}{4 \pi} \sqrt{1-\frac{1}{N_{c}}} \frac{1}{r+r_{\phi}}+\tilde{c}$,

where $\tilde{c}$ is an integration constant. The potential energy is given by

$u_{c l}(r)=q v_{c l}(r)$.
Expressing the charge $q$ in terms of $g$ as defined above, we found

$u_{c l}(r)=-\epsilon_{0} g^{2}\left(1-\frac{1}{N_{c}}\right) \frac{1}{4 \pi\left(r+r_{\phi}\right)}$

where for simplicity we also have here disregarded the integration constant and in Eq. (57), Eq. (43) has been used. The Coulomb-type potential energy for the electric field mimics the energy of quarks and gluons in the limit of high energies as $r \rightarrow 0$. Note, however, that the potential is regular at $r=0$ unlike a usual Coulomb potential. The confining and Coulomb-like potentials are depicted in Fig. 1. Note that the numerical solution $v_{n}$ (depicted in red) smoothly connects these two regimes in small and large distances. Here we solve numerically Eq. (37) for $\alpha=0.01$ and $\lambda=1$. With this, we use $g / 4 \pi=1, N_{c} \gg 1, r_{\phi}^{2}=(0.05)^{-1}$, and $\tilde{c}=20$ in potentials $v_{c}$ and $v_{c l}$. As expected, at small distances the potential approaches the confining solution $v_{c}$ (consider $c=0$ ) Fig. 1-top-and at large distances the potential approaches a constant given by $v_{c l}$ (i.e., $\tilde{c}=2 m_{q}$ )-Fig. 1-bottom. This is the regime where hadronization processes take place via the production of light mesons, i.e., for small masses $m_{q}$.

Finally, we shall comment on the relation between the confinement and the tachyon condensation. We will also comment on the connection in between the confinement and deconfinement phases at small and large distances presented numerically.

We have found the linear confining by assuming $\alpha \phi$ sufficiently large (i.e., $r \gg r_{\phi}$ ) in Eq. (37) that leads to Eq. (38), which for the tachyon potential

$V=e^{-\alpha \phi}$

presents the explicit confining solution (40). When we substitute this solution into the tachyon potential we find

$V(r)=e^{-\alpha \phi(r)}=\left(\frac{r_{\phi}}{r}\right)^{2}$.

We note from (59) that the tachyon condensation, i.e., $V \rightarrow$ 0 , happens when $r / r_{\phi} \gg 1$. This is automatically satisfied by the hypothesis that $r \gg r_{\phi}$.

On the other hand, from the numerical analysis, since the dielectric function $G(r) \equiv V(r)$, Fig. 2 suggests that the confinement/condensation condition is again easily satisfied at $r \simeq 100$ since we have chosen $r_{\phi}^{2}=(0.05)^{-1}$. We can therefore conclude that the electric confinement is associated with tachyon condensation. From the 't Hooft-Mandelstam and Seiberg-Witten [11-13] theories, which deal with the duality between the phenomena of confinement and superconductivity, we know that the electric confinement can also be associated with the condensation of magnetic monopoles. However, both isolated tachyons and magnetic monopoles have never been observed. 

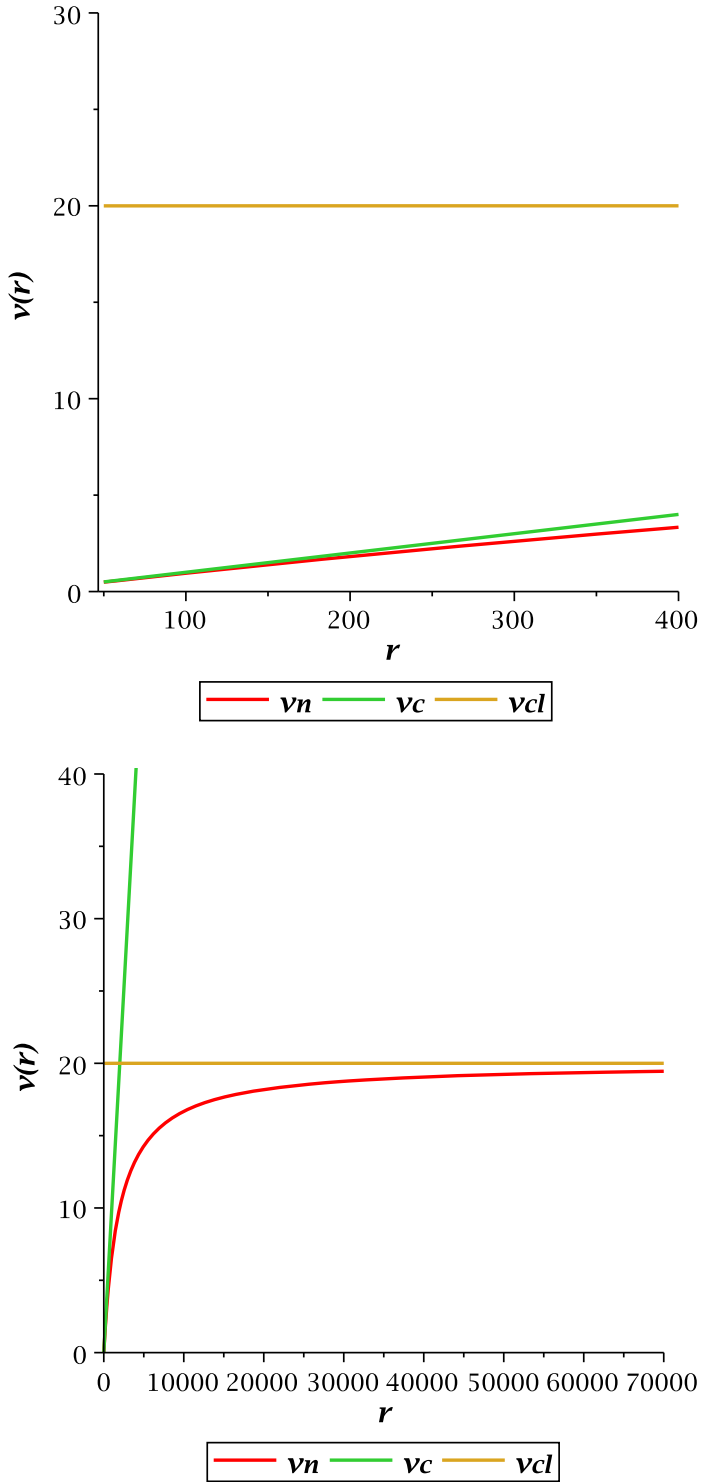

Fig. 1 The numerical solution $v_{n}$ smoothly connects the two regimes in small $\left(v_{c}\right)$ and large $\left(v_{c l}\right)$ distances. (top) Potentials at small distances: $v_{n}$ approaches $v_{c}$ at a linear confinement phase. (bottom) Potentials for large distances: $v_{n}$ approaches $v_{c l}$ at a deconfinement (or hadronization) phase

Now we focus our attention to the deconfining regime. Following a similar analysis and hypothesis using the nonconfining solution (52) we found

$V(r)=\left(\frac{r+r_{\phi}}{r}\right)^{2} \rightarrow 1$

Again, since $G(r) \equiv V(r)$, Fig. 2 suggests that the nonconfining regime at sufficiently large distance does not correspond to tachyon condensation. Instead, the full numeric solution depicted in Fig. 1 indeed allows us to identify this regime with a hadronization phase.

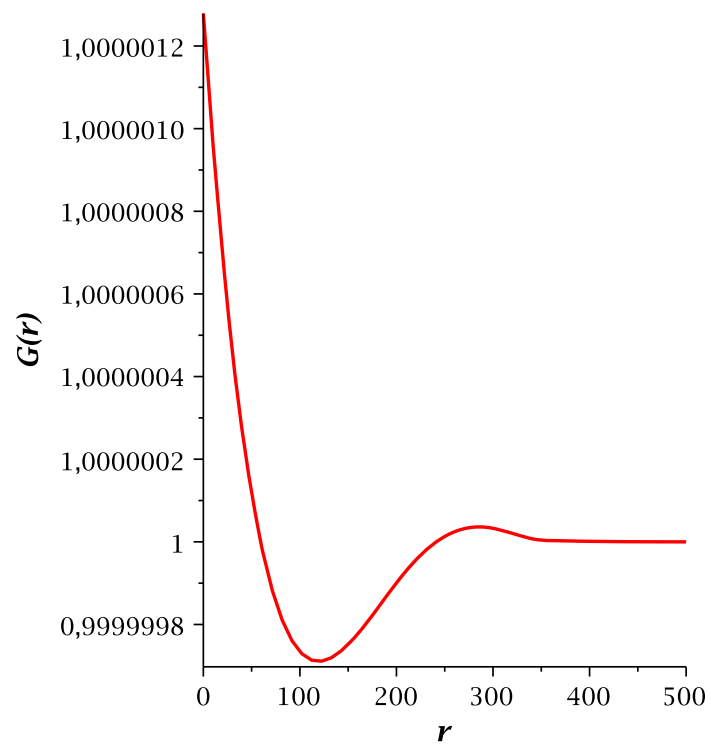

Fig. 2 The numerical solution for the dielectric function: $G(r)$ develops a decreasing behavior until $r \simeq 100$ showing a confining (antiscreening) phase and then it increases with $r$ developing a deconfining (screening) phase

Our numerical solution shows that there is a QCD-like string breaking such that the confining is linear only in a certain range, Fig. 1 (top), and then the potential becomes constant, which is typical of a hadronization phase.

As a last remark one should note that a large typical hadron size $r_{\phi} \sim 1 / m_{q}$, which means the limit $m_{q} \rightarrow 0$ (light mesons), favors a regime in which a screening phenomenon after the confining phase comes into play, because the QCD string tension given in (48) becomes small. Then it is expected to be broken more easily than for heavy mesons. Thus, for light quarks QCD is never truly confining [20].

\section{Conclusions}

In this study we found a Coulomb-like and confinement potential to the electric field that resembles the ones obtained for the quarks and gluons. Our Abelian approach can be understood as an approximation of the non-Abelian QCD theory. The confining/deconfining regime of the electric field was obtained considering a dielectric medium whose tachyon dielectric function describes an anti-screening/screening behavior at small and large distance. This medium is regarded as a tachyon matter described in terms of a tachyon potential that vanishes at the minima, that is, in the tachyon condensation. Tachyon condensation was shown to be related to the electric confinement. Thus the tachyon condensation plays the same role as the condensation of monopoles in the electric confinement phenomenon. The latter corresponds to duality between the confinement and the phenomenon of superconductivity. 
Acknowledgments We would like to thank CNPq, CAPES, PROCADCAPES for partial financial support. We also thank Tiago Mariz and Emanuel Cunha for discussions.

Open Access This article is distributed under the terms of the Creative Commons Attribution License which permits any use, distribution, and reproduction in any medium, provided the original author(s) and the source are credited.

Funded by $\mathrm{SCOAP}^{3}$ / License Version CC BY 4.0.

\section{References}

1. E. Eichten et al., Phys. Rev. Lett. 34, 369 (1975)

2. L. Wilets, Nontopoloeical Solutions (Word Scientific, Singapore, 1998)

3. T.D. Lee, Particles Physics and Introduction to Field Theory (Harwood Academic, New York, 1981)

4. H. Shiba et al., Phys. Lett. B 333, 461 (1994)

5. G.S. Bali et al., Phys. Rev. D 54, 2863 (1996)

6. A. Chodos, R.L. Jaffe, K. Jonhson, C.B. Thorn, V.F. Weisskopf. Phys. Rev. D 9, 3471 (1974)
7. W.A. Bardeen, M.S. Chanowitz, S.D. Drell, M. Weinstein, T.-M. Yan, Phys. Rev. D 11, 1094 (1975)

8. R. Friedberg, T.D. Lee, Phys. Rev. D 15, 1964 (1997)

9. A. Sen, JHEP 0207, 065 (2002). arXiv:hep-th/0203265

10. A. Sen, JHEP 0204, 048 (2002). arXiv:hep-th/0203211

11. S. Mandelstam, Phys. Rep. 23C, 145 (1976)

12. G. 't Hooft, in Proceed. of Euro. Phys. Soc. 1975, ed. A. Zichichi

13. N. Seiberg, E. Witten, Nucl. Phys. B 426, 19 (1994)

14. M. Cvetic, A.A. Tseytlin, Nucl. Phys. B 416, 137 (1994)

15. B. Zwiebach, String Theory (University Press, Cambridge, 2004)

16. D. Bazeia, F.A. Brito, W. Freire, R.F. Ribeiro, Int. J. Mod. Phys. A 18, 5627 (2003)

17. D. Bazeia, F.A. Brito, W. Freire, R.F. Ribeiro, Eur. Phys. J. C 40, 531 (2005). arXiv:hep-th/0311160

18. R. Dick, Phys. Lett. B 409, 321 (1997)

19. R. Dick, Eur. Phys. J. C 6(4), 701-703 (1999)

20. M.J. Strassler, On confinement and duality, in Superstrings and Related Matters Trieste, 2001, pp. 105-162 